\section{Nanoscopy with more than 100,000 'doughnuts'}

\author{
Andriy Chmyrov ${ }^{1}$, Jan Keller ${ }^{1}$, Tim Grotjohann ${ }^{1}$, \\ Michael Ratz ${ }^{1}$, Elisa d'Este ${ }^{1}$, Stefan Jakobs ${ }^{1,2}$, \\ Christian Eggeling ${ }^{1} \&$ Stefan W Hell ${ }^{1}$
}

\begin{abstract}
We show that nanoscopy based on the principle called RESOLFT (reversible saturable optical fluorescence transitions) or nonlinear structured illumination can be effectively parallelized using two incoherently superimposed orthogonal standing light waves. The intensity minima of the resulting pattern act as 'doughnuts', providing isotropic resolution in the focal plane and making pattern rotation redundant. We super-resolved living cells in $120 \mu \mathrm{m} \times 100 \mu \mathrm{m}$-sized fields of view in $<1$ s using 116,000 such doughnuts.
\end{abstract}

Super-resolution far-field fluorescence microscopy or nanoscopy ${ }^{1-4}$ typically discerns features residing closer than the diffraction barrier $D$ of $\sim 250 \mathrm{~nm}$ by making their fluorophores transiently assume different states ${ }^{1,5}$. These are usually molecular 'on' and 'off' states between which fluorophores differ in their ability to emit fluorescence. Whereas stochastic super-resolution approaches ${ }^{2-4}$ induce on-state molecules individually and randomly in space, the coordinate-targeted counterparts of these techniques-such as stimulated emission depletion (STED) ${ }^{6}$, saturated patterned excitation microscopy (SPEM) or saturated structured illumination microscopy (SSIM) $)^{7,8}$, and RESOLFT ${ }^{1,9}$-induce the on and off states at precisely defined positions in the sample ${ }^{5}$. For this purpose, such approaches apply a pattern of light with intensity zeros, typically a doughnut ${ }^{1,5}$ or a standing wave ${ }^{7,8}$, which drives all fluorophores briefly to one of these states except for those fluorophores located within a narrow range of extent $d \approx D /\left(1+I / I_{\mathrm{s}}\right)^{1 / 2}<D$ around the zeros ${ }^{1,5}$. Thus, for a short detection period, features from within this subdiffraction-sized space can be discerned by their state, for example, by being 'on' (fluorescent) when their neighbors are 'off', or vice versa. $I_{\mathrm{s}}$ is a parameter giving the light intensity at which the probability of changing the state is $50 \%$. $I>I_{\mathrm{s}}$ is the peak intensity bordering the zero. Scanning the pattern across the sample and measuring the fluorescence signal with a step (pixel) size $<d / 2$ fulfilling the Nyquist condition produces image data with subdiffraction resolution $d$ (refs. 1,5).

Such coordinate-targeted methods can provide imaging at video rates and faster ${ }^{10}$, but their recording speed is still governed by the kinetics of the state transition and, most prominently, by the number of scanning steps (pixels) required for covering the field of view. Unfortunately, if large fields are to be imaged with just a single zero (for example, one doughnut), the number of steps can be exceedingly large. This problem can be addressed by developing parallelized scanning schemes applying arrays of zeros with regular spacings $P \geq D$, where $D \approx 250 \mathrm{~nm}$ (refs. $1,7,8$ ). In this case, the fluorescence generated at each zero can be identified on a camera and read out for each scanning step. Parallelized scanning thus reduces the number of steps to the number $F=(2 p / d)^{2}$ required to scan each of the zeros over the diffraction area $p \times p \approx D \times D \approx(250 \mathrm{~nm})^{2}$. For example, for $d=50 \mathrm{~nm}, F=100$ steps are sufficient to super-resolve any field of view.

Parallelized fluorescence nanoscopy was first demonstrated as $\mathrm{SSIM}^{8}$, whereby a one-dimensional (1D) standing-wave pattern of intense $\left(I>I_{\mathrm{s}} \approx 1-10 \mathrm{MW} \mathrm{cm}^{-2}\right)$ excitation light forced all fluorophores to their fluorescent (on) state except for those within the subdiffraction range $d<<D$ around the line-shaped zeros, where they remained off. SSIM requires data processing because the information is contained in the dark off-state region ('negative imaging') and because the scanning standing wave has to be rotated by $(180 / m)^{\circ}$, with the number of rotations $m \approx 9-12$, and $m$ increases beyond 12 with decreasing $d$ (ref. 8). The large intensities also cause photobleaching, and the mathematical processing of the negative data sets is challenging because of noise. Alternatively, one can perform 'positive imaging' by creating $d$-sized on-state regions ${ }^{1,5}$ through STED using multiple circular doughnuts ${ }^{11}$. This scheme yields raw data images, but the required intensities are as large as for SSIM ${ }^{1,5}$, which is why the number of doughnuts implemented so far in parallel has been limited to four ${ }^{11}$.

Clearly, coordinate-targeted nanoscopy of large fields is preferably realized with fluorophores that require much lower intensities $I_{\mathrm{s}}$, such as reversibly switchable fluorescent proteins (rsFPs) ${ }^{1,5,9}$. This approach, known as RESOLFT, has recently been used to super-resolve living cells and tissues ${ }^{12-14}$ at light intensities $\sim 10^{5}$ times lower than those used for SSIM and STED. However, the initial implementations of RESOLFT were performed with only a single doughnut beam, which, owing to the relatively slow switching kinetics of the rsFPs used, led to recording times of 20-30 min in $\sim 10-\mu \mathrm{m}$ fields $s^{9,12,13}$. More recent rsFPs helped reduce the recording time to fractions of a minute ${ }^{15}$. Parallelization of rsFP-based RESOLFT nanoscopy using a 1D standing-wave pattern was demonstrated as well ${ }^{16,17}$; however, these demonstrations involved rotating the pattern as in SSIM, and the recording was still slow ${ }^{17}$.

Here we demonstrate that the inverse-square-root dependence of $d$ on the applied intensity ${ }^{1,5}$ reflected in the $d \approx D /\left(1+I / I_{\mathrm{s}}\right)^{1 / 2}$ equation enables an elegant method for parallelizing all forms of coordinate-targeted nanoscopy; this parallelization provides

${ }^{1}$ Department of NanoBiophotonics, Max Planck Institute for Biophysical Chemistry, Göttingen, Germany. ${ }^{2}$ Department of Neurology, University of Göttingen, Göttingen, Germany. Correspondence should be addressed to S.W.H. (shell@gwdg.de). 

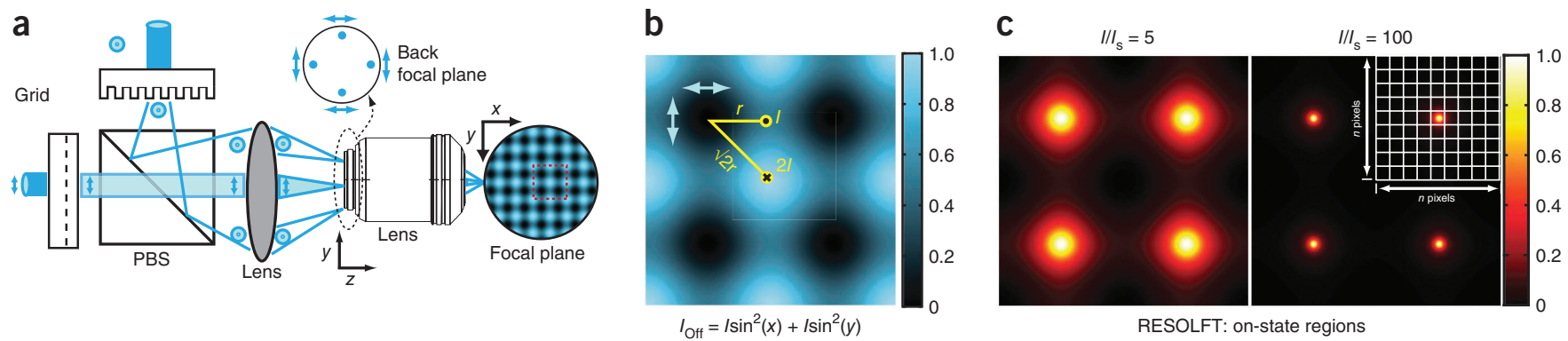

Figure 1 | Parallelized scanning (RESOLFT) nanoscopy using orthogonally and incoherently crossed standing waves. (a) Generation of a dense pattern of zero-intensity points in the focal plane of an objective lens: two beams pass diffraction gratings oriented parallel to the polarization of the incident beam. Light of the first diffraction orders is recombined with a polarizing beam splitter cube (PBS) and focused to the entrance pupil of the lens. Collimated in the focal plane, the beams generate a pattern consisting of the incoherent addition of two orthogonal sinusoidal standing light waves. At the intersections of their valleys and crests, the standing waves form zero points and peaks of twice the crest intensity, respectively. The blue arrows indicate the polarization. The diffracted beams transmitted by the PBS propagate within the drawing plane, whereas the reflected beams travel orthogonally to it. (b) Zoomed-in view of the $2 \mathrm{D}$ intensity profile of the off-switching pattern shown in a. $I$ is the maximum intensity at the crest of a single standing wave and $r$ is the radial coordinate around a zero-intensity point. (c) 2D profiles of the on-state probability distribution for different $I / I_{s}$, where $I_{s}$ is the light intensity at which the state transfer probability is $50 \%$. The fine grid represents the scanning range.

isotropic resolution in the focal plane and makes pattern rotation redundant. As a result, raw super-resolution images are gained at maximum degree of parallelization $(p \approx D)$.

This concept reconciles major goals of super-resolution development: low-intensity operation, large fields of view and fast recording, at a resolution that is conceptually diffraction unlimited. The power of this strategy is demonstrated with a $2 \mathrm{D}$ array of $>110,000$ intensity zeros acting as doughnuts and providing isotropic super-resolution in parallel. The recording speed is limited by only the state transition kinetics of the fluorophore and the camera frame rate.

We used rsFPs that are switched on at wavelength $\lambda=405 \mathrm{~nm}$ and off at $\lambda=488 \mathrm{~nm}$ (blue). The same blue light also elicits fluorescence that is detected in the 500- to $580-\mathrm{nm}$ range. To obtain raw data images, we implemented RESOLFT in the 'positive imaging' mode, meaning that an off-switching pattern of blue light was used to create $d$-sized regions of on-state fluorophores. These on-state rsFPs were imaged onto a camera by wide-field blue excitation, which also switched them off. The cycle was completed by wide-field on-switching at $\lambda=405 \mathrm{~nm}$ and was repeated for each scanning step.

Our off-switching pattern is strikingly simple: two orthogonally and incoherently crossed standing waves (ICSs) producing zero-intensity points at the intersections of their zero-intensity lines (Fig. 1). Because each standing wave improves the resolution along only the $x$ or $y$ direction, one would expect the ICS pattern to require rotation to cover other directions as well, particularly along the diagonals. Although rotation is certainly needed for moderate resolution improvements $\left(I<I_{\mathrm{s}}\right)$ as found in 'linear' structured illumination ${ }^{18,19}$, for the larger intensities providing nanoscale imaging $\left(I>5 I_{\mathrm{s}}\right)$, the resolution becomes increasingly isotropic (Fig. 1c), making rotation redundant. Already for $I=5 I_{\mathrm{s}}$ the spatial distribution of the on-state in the focal plane exhibits nearly circular full-width at half-maximum (FWHM) contour lines (Fig. 1b). For a tenfold and higher improvement-that is, $I>100 I_{\mathrm{s}}$-the resolution is essentially isotropic (Fig. 1c).

This arguably surprising outcome traces back to the $d \approx D /$ $\left(1+I / I_{\mathrm{s}}\right)^{1 / 2}$ law $^{1,5}$. Whereas the distance from the zero point to the nearest intensity peak is larger by $\sqrt{ } 2$ along the diagonals than in the $x$ and $y$ directions, the diagonal peaks are twice as high, causing the inverse-square-root dependence on the intensity to neutralize the larger distance (Fig. 1b). Expanding the intensity minimum with a 2D Taylor series also reveals that the intensity scales quadratically with the distance from the zero, confirming the circular symmetry of the minimum around the zero points (Supplementary Note 1). For $I \gg I_{s}$, only the regions around the minima matter, meaning that the minima of the ICS pattern act as doughnuts.

Pertinent to this finding is the fact that standing waves inherently provide the steepest intensity minima at the highest spatial density, that is, at a minimal distance $P=\lambda /(2 n \sin \theta) ; n$ denotes the refractive index and $\theta$ is the angle formed by the plane waves with the optic axis $z$. We used a 1.46-numerical aperture (NA) oil-immersion lens, and $\theta=30^{\circ}$ so that our 2D doughnut array featured $P=320 \mathrm{~nm}$ and $I \approx 1 \mathrm{~kW} \mathrm{~cm}^{-2}$ at the single standing-wave peak. The peaks in the ICS pattern border two or four minima in parallel, which also lowers the total light dose as compared to circular doughnut arrays.

We recorded the images by scanning the sample with respect to the stationary array of zeros while detecting the fluorescence with $1,200 \times 1,040$ quadratic pixels of a scientific complementary metal-oxide semiconductor (sCMOS) camera; these pixels were $98 \mathrm{~nm}$ in size when imaged down to the focal plane. Thus, the zeros were associated with pixels functioning as pinholes of about one-fifth of the Airy disc. This arrangement exhibits quasiconfocal axial resolution because fluorescence originating from $d$-sized regions above and beneath the focal plane is blurred at the camera and can be subtracted (Supplementary Note 2 and Supplementary Figs. 1 and 2). The $~ 580$-nm optical sectioning obtained with this system also suppresses signal from fluorophores that are laterally away from the zero, thus further enhancing the isotropic emission geometry around the zero point.

All together, we generated $366 \times 317=116,022$ zeros in parallel. We applied them to RESOLFT nanoscopy (Fig. 2 and Supplementary Fig. 3), imaging filaments of keratin 19 fused to the novel rsEGFP variant rsEGFP(N205S) (Supplementary Figs. 4-8) in living PtK2 cells at a resolution of $\sim 80 \mathrm{~nm}$ (FWHM). rsEGFP(N205S) switches 2-5 times slower than the reported 
a

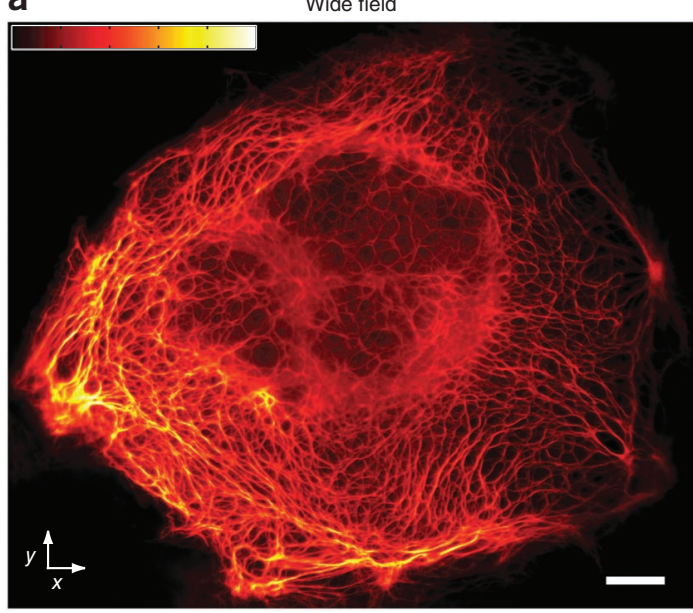

C

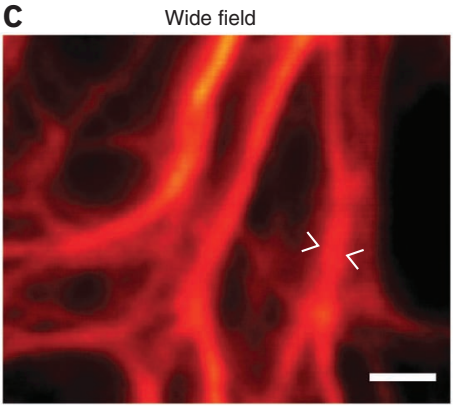

b
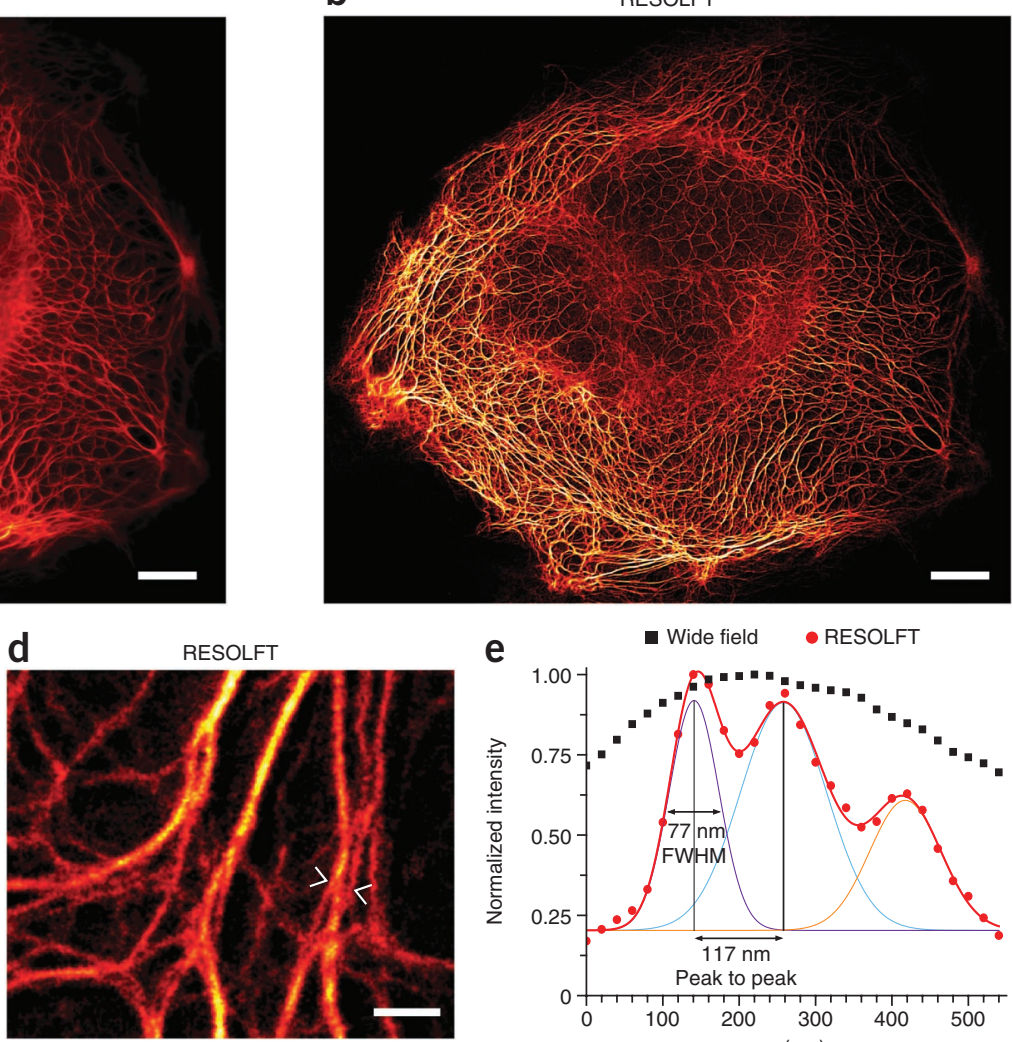

- Wide field RESOLFT

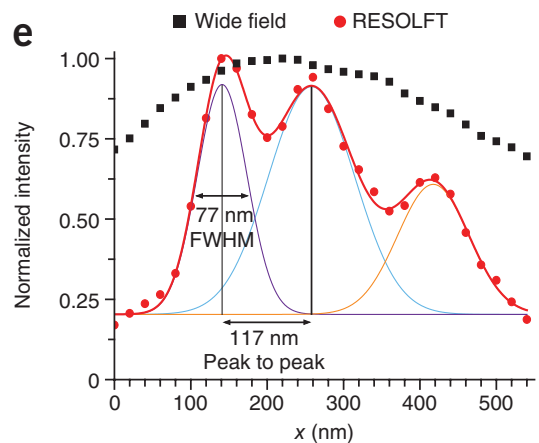

Figure 2 | Live-cell imaging with parallelized RESOLFT nanoscopy. (a,b) The $120 \mu \mathrm{m} \times 100 \mu \mathrm{m}$ field of view (wide field (a) and RESOLFT (b)) shows PtK2 cells expressing keratin 19-rsEGFP(N205S). The RESOLFT image was reconstructed from 144 frames, each acquired in 22 ms; total image acquisition time was $\sim 3$ s. Scale bars, $10 \mu \mathrm{m}$. Intensity is from black, low, to white, high. (c,d) Magnified region (wide field (c) and RES0LFT (d)) of a PtK2 cell expressing keratin 19-rsEGFP(N205S) (Supplementary Fig. 3). Scale bars, $1 \mu \mathrm{m}$. (e) Normalized intensity profiles of the regions between the arrowheads in c (black squares) and $\mathbf{d}$ (red dots). The profile of the RESOLFT data (red line) is fitted to a sum of three Gaussians (purple, green and orange lines) with individual full-width half-maxima (FWHM) of $77 \mathrm{~nm}, 133 \mathrm{~nm}$ and $110 \mathrm{~nm}$.

rsEGFP $^{12}$ at the light intensities used. However, by emitting about twice the number of photons per cycle, rsEGFP(N205S) provides an improved signal-to-noise ratio in each camera frame, facilitating camera background subtraction. Apart from background subtraction, the images and the resolution value are raw, that is, obtained without deconvolution. The step (pixel) size $\kappa$ was $26.6 \mathrm{~nm}$, meaning that $F=(p / \kappa)^{2}=(320 / 26.6)^{2}=144$ steps were enough to (over)sample the $120 \mu \mathrm{m} \times 100 \mu \mathrm{m}$-sized field of view. Recording was accomplished within $2-4 \mathrm{~s}$, with speed determined by the switching kinetics of rsEGFP(N205S). The faster switching of $\operatorname{rsEGFP}^{12}$ and the rsFP Dronpa-M159T ${ }^{14}$ allowed

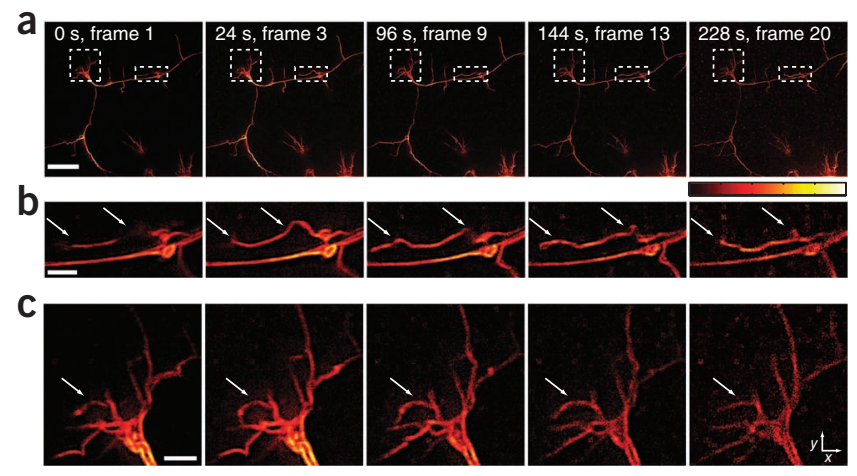

us to image the same field of view within $1 \mathrm{~s}$ (Supplementary Fig. 9), limited by the camera readout time. Consequently, imaging only $50 \mu \mathrm{m} \times 50 \mu \mathrm{m}$ cut down the recording time to $400 \mathrm{~ms}$ (Supplementary Fig. 10). We also performed sequential RESOLFT imaging of an outgrowing neurite (Fig. 3).

The focal-plane resolution is not affected by our parallelization scheme. For large enough $I / I_{s}$, the resolution $d$ can theoretically attain molecular scales. However, in practice the resolution is also determined by the number of on-off cycles possible before bleaching. Because the fluorophores undergo a full cycle for each of the $F$ scanning steps, $F$ cycles allow a Nyquist-compatible pixel size of $d / 2=p / \sqrt{ } F$. Using $p \approx D$, we find that each fluorophore has to accommodate at least $F \approx 4 I / I_{\mathrm{s}}$ cycles. Intermediate states involved in the switching of rsFP and the associated state kinetics

Figure 3 | Continuous time-lapse imaging with parallelized RESOLFT. (a) Frames of a time series of outgrowing neurites and filopodia-like structures of a living neuronal cell expressing Lifeact-Dronpa-M159T fusion protein. (b,c) Enlargements of right (b) and left (c) marked regions in $\mathbf{a}$. So that the small filaments are highlighted over the thick and bright fibers, the images are displayed using a nonlinear intensity scale ( $\gamma=0.5$; black, low, to white, high). Individual RESOLFT images from the time series were recorded in $2 \mathrm{~s}$. The arrows indicate structures that moved in and out of the focal plane. Scale bars, $10 \mu \mathrm{m}(\mathbf{a})$ and $2 \mu \mathrm{m}(\mathbf{b}, \mathbf{c})$. 
cause $F$ to also depend on absolute values of $I$ and the pixel dwell times. Therefore, the parameters chosen in this study are not exclusive: another combination may provide higher resolution or faster imaging speed.

Speed limits set by the switching kinetics may also be overcome with new rsFPs. Yet protein switching is just one possible on-off transition; our ICS scheme applies to all coordinate-targeted methods, including STED. As STED entails ultrafast on-off switching, implementing the ICS pattern with STED should provide movies limited in speed by only the rate of photon registration.

We exerted our $p / \kappa=144$ scanning steps with a simple piezostage, whereas approaches based on 1D standing-wave patterns usually use spatial light modulators or similar devices to perform pattern scanning and rotation. Pattern rotation not only adds complexity and data processing but, because rotation typically takes longer than making a few hundred steps, also slows down recording. Conversely, scanning with a rotating pattern requires fewer on-off cycles, namely, $m \times 2 p / d<(2 p / d)^{2}$. However, as the number of rotations $m$ increases with smaller $d$ (ref. 17), this advantage is lost, and the need for deconvolution with a known point-spread function remains.

Another conceptual strength of the ICS pattern is that the intensity minima are truly 0 : deviations are solely due to practical imperfections such as aberrations and scattering (Supplementary Fig. 11). In contrast, the slopes of the minima have a polarization bias due to the linear polarization of the standing waves, which causes the efficiency of the state transition to vary with the orientation of the molecules in space. Fortunately, coordinate-targeted methods are less prone to polarization effects than their stochastic single-molecule counterparts because the signal from a given pixel typically stems from many randomly oriented fluorophores. These effects are further reduced by rotational diffusion within the $\sim 1$ - to 5-ms acquisition time of a single frame. In refinements of our scheme, circularly polarized standing waves may be adapted. Also, the axial resolution can be improved by coherently adding a plane-parallel wave propagating along the $z$ axis $^{20}$.

Unlike single-doughnut arrangements, our approach needs no alignment with the activation and excitation beams. Drifts will shift the pattern only with respect to the camera sensor. Besides, the $<0.5$ - to 2 -s total imaging time makes long-term stability unnecessary. Stability is more of a concern in rotating pattern schemes because the same features need to be scanned several times and the data co-registered for mathematical processing. The ICS pattern is equally useful for the 'negative imaging' modalityin which the pattern switches fluorophores on, creating $d$-sized off-state regions-such as in SSIM. Although data processing is inevitable in this case, rotation is still avoided.

Parallelized forms of coordinate-targeted super-resolution microscopy are frequently modeled in frequency space using Moiré patterns ${ }^{1,5}$. Our study illustrates the simplicity and power of real-space explanations. Likewise, the use of large-field illumination, camera detection and switchable fluorescent proteins highlights similarities between coordinate-targeted and stochastic methods. Although both nanoscopy families are ultimately limited by the recurrence of two states, the contrast between these states and the sensitivity of state detection, a major difference remains: whereas the stochastic methods localize fluorophores using photons originating from the sample, their coordinatetargeted counterparts 'localize' the fluorophores with a photon pattern incident upon the sample. The ICS pattern is a versatile photon pattern, irrespective of the state transition used. Simple, easy to implement and containing densely packed zeros that yield nearly isotropic resolution, this concept should become a standard in super-resolution microscopy. Finally, as the parallelization degree depends on the available illumination power, the number of doughnuts can be expanded to millions.

\section{METHODS}

Methods and any associated references are available in the online version of the paper.

Note: Supplementary information is available in the online version of the paper.

\section{ACKNOWLEDGMENTS}

M. Leutenegger (formerly at Max Planck Institute for Biophysical Chemistry, Göttingen; now at the Ecole Polytechnique Fédérale de Lausanne) is acknowledged for early discussions about technical aspects. A.C. acknowledges a postdoctoral scholarship from the Swedish Research Council. S.J. and S.W.H. acknowledge funding from the German Research Foundation (DFG)-Center of Nanoscale Microscopy and Molecular Physiology of the Brain. S.W.H. also received support from the German Ministry of Research (BMBF) and the Körber Foundation, Hamburg, through the European Science Prize.

\section{AUTHOR CONTRIBUTIONS}

A.C. built the setup, performed the experiments and analyzed data. J.K. analyzed data and performed data modeling. T.G. developed and characterized the protein rsEGFP(N205S), advised by S.J. M.R. and E.d'E. prepared samples. C.E. advised on protein switching and setup implementations. S.W.H. defined and supervised the project, wrote the paper and is, together with A.C., responsible for its main thrust.

\section{COMPETING FINANCIAL INTERESTS}

The authors declare competing financial interests: details are available in the online version of the paper.

Reprints and permissions information is available online at http://www.nature. com/reprints/index.html.

1. Hell, S.W. Nat. Biotechnol. 21, 1347-1355 (2003).

2. Rust, M.J., Bates, M. \& Zhuang, X.W. Nat. Methods 3, 793-795 (2006).

3. Betzig, E. et al. Science 313, 1642-1645 (2006).

4. Hess, S.T., Girirajan, T.P.K. \& Mason, M.D. Biophys. J. 91, 4258-4272 (2006).

5. Hell, S.W. Nat. Methods 6, 24-32 (2009).

6. Hell, S.W. \& Wichmann, J. Opt. Lett. 19, 780-782 (1994).

7. Heintzmann, R., Jovin, T.M. \& Cremer, C. J. Opt. Soc. Am. A Opt. Image Sci. Vis. 19, 1599-1609 (2002).

8. Gustafsson, M.G.L. Proc. Natl. Acad. Sci. USA 102, 13081-13086 (2005).

9. Hofmann, M., Eggeling, C., Jakobs, S. \& Hell, S.W. Proc. Natl. Acad. Sci. USA 102, 17565-17569 (2005)

10. Westphal, V. et al. Science 320, 246-249 (2008).

11. Bingen, P., Reuss, M., Engelhardt, J. \& Hell, S.W. Opt. Express 19, 23716-23726 (2011).

12. Grotjohann, T. et al. Nature 478, 204-208 (2011).

13. Brakemann, T. et al. Nat. Biotechnol. 29, 942-947 (2011).

14. Testa, I. et al. Neuron 75, 992-1000 (2012).

15. Grotjohann, T. et al. elife 1, e00248 (2012).

16. Schwentker, M.A. et al. Microsc. Res. Tech. 70, 269-280 (2007).

17. Rego, E.H. et al. Proc. Natl. Acad. Sci. USA 109, E135-E143 (2012).

18. Gustafsson, M.G.L. J. Microsc. 198, 82-87 (2000).

19. Frohn, J.T., Knapp, H.F. \& Stemmer, A. Proc. Natl. Acad. Sci. USA 97 7232-7236 (2000).

20. Gustafsson, M.G.L. et al. Biophys. J. 94, 4957-4970 (2008). 


\section{ONLINE METHODS}

Setup. Our parallelized RESOLFT microscope uses a beam for wide-field on-switching (activation) of rsFPs, four beams to generate the off-switching pattern plus another beam for uniform wide-field readout. Two continuous-wave diode lasers from Omicron-Laserage Laserprodukte were used, one for activation (LuxX 405-120, $405 \mathrm{~nm}, 120 \mathrm{~mW}$ ) and another one for fluorescence off-switching and excitation (LuxX 488-200, 488 nm $200 \mathrm{~mW}$ ). The latter was spectrally cleaned with an excitation filter (BrightLine 488/6), sent through a Glan-Thomson polarizer, an electro-optical modulator (EOM, LINOS LM0202P5W, Qioptiq) and a polarizing beam splitter (PBS). By applying a TTL signal to the EOM driver, the beam was switched between switch-off (reflected) and excitation (transmitted) paths with high extinction ratio $(1: 1,400)$. The $488-\mathrm{nm}$ switch-off light was coupled into a single mode polarization-maintaining PC/APC fiber (P5-488PMFC-2, Thorlabs) using the coupler 60FC-4-4.5S-01 (Schäfter + Kirchhoff). The light exiting the fiber was collimated with an achromatic doublet (focal length $f=40 \mathrm{~mm}$ ) and directed through a half-wave plate and a PBS. The orientation of the half-wave plate was adjusted such that the light was equally divided by the PBS. After the PBS, the light was sent through custom-made phasediffraction gratings of 437-nm-high $\mathrm{SiO}_{2}$ lines with a $36-\mu \mathrm{m}$ period (Laser Laboratorium Göttingen). The gratings were oriented such that the grid lines were parallel to the polarization of the incoming light. After the gratings, the light paths were recombined with another PBS. Using a lens of $100-\mathrm{mm}$ focal length, the diffracted light was focused to a secondary pupil, and all of the diffraction orders except +1 and -1 were blocked; these orders were relayed by a pair of lenses $(f=200 \mathrm{~mm})$ to four spots in the back focal plane (BFP) of a $100 \times / 1.46-\mathrm{NA}$ objective lens (Olympus), where they recombined and created a regular sinusoidal pattern in two orientations with a period of $320 \mathrm{~nm}$. The gratings were manually rotated until orthogonality of the patterns was achieved. By varying the focal length of the lens after the diffraction gratings, it was possible to vary the position of the diffracted orders in the back aperture (here at $48 \%$ of the radius), which in turn varied the period of the pattern. The excitation light was expanded by a telescope and combined with the deactivation light using a 50:50 beam splitter. The on-switching (activation) light was expanded by a telescope and combined with the excitation and off-switching light by a dichroic mirror Z405RDC. The fluorescence was collected by the same objective lens and separated by a dichroic mirror 505DCXRU, filtered using a fluorescence filter BrightLine HC525/50, directed through a tube lens $(f=120 \mathrm{~mm})$ and imaged onto a 5-megapixel sCMOS camera (Andor Neo), which was cooled to $-40^{\circ} \mathrm{C}$. Usually only a small centered subarray of the total $1,392 \times 1,040$ pixels was read out from the camera. A flat-field correction of the camera frames was applied as described in Supplementary Note 3. The focal length of the tube lens was chosen in such a way that the $6.5-\mu \mathrm{m}$ pixels on the camera projected to $98-\mathrm{nm}$ pixels in sample space. Scanning of the sample was achieved using a three-axis piezo stage NanoMax (Thorlabs). A detailed scheme of the setup is depicted in Supplementary Figure 12. The polarization optics and beam splitters were from B. Halle Nachfl. The fluorescence filters were from Semrock. The dichroic mirrors were made on a thick 6-mm substrate (Chroma). The lenses were achromatic doublets (Qioptiq).
Image acquisition and reconstruction. The custom image acquisition and analysis software were written using Matlab. A multifunction analog output computer card NI PCI-6731 (National Instruments) was used to trigger the lasers and the EOM, to adjust the power of the 488-nm laser and to control the position of the stage. The power of the 405-nm laser was controlled via the USB port. The camera fire pulse was used to trigger the image acquisition sequence. For the acquisition of a full RESOLFT image, the sample was shifted by the piezo stage typically ten times in each direction, which resulted in a stack of 100 camera images. Each camera image was a result of three successive illuminations: switching (activation) of all molecules to the on state with uniform illumination at $405 \mathrm{~nm}$, off-switching (deactivation) of a subset of molecules to the off state with the orthogonally crossed pattern at $488 \mathrm{~nm}$ and excitation of the remaining on-state molecules with uniform illumination at $488 \mathrm{~nm}$. After completing this cycle, the sample was shifted to a new position. The pattern was stationary with regard to the camera. To achieve higher time efficiency, we carried out the sample movement and the on-switching pulse simultaneously. The recorded stack of camera images was summed up, and the period and the offset in each direction of the stationary off-switching pattern was identified by correlation of the data to a regular 2D sinusoidal profile, i.e., the sum of sinusoidal stripes in $x$ and $y$.

Owing to degradation or insufficient activation of the fluorescent proteins, the total fluorescence intensity of each camera frame decreased slightly after each recording. We corrected for this by normalizing to a fixed total intensity per frame. Through the use of identified pattern parameters, each camera frame was interpolated to a finer grid (typically $20 \mathrm{~nm}$ and descanned, i.e., the origin was fixed in object space), and then a positive weighting function (Gaussian with 50-nm width) was applied to the minima positions and also to the surrounding maxima positions separately. The interpolated, descanned and weighted camera frames were added and resulted in an image consisting of the minima position values containing the high-resolution data and an image consisting of the maxima position values containing local background. A background-reduced image was obtained by subtracting $80 \%$ of the image containing the values from the positions of the minima from the image containing the values from the positions of the maxima and setting the remaining negative values to 0 . No a priori knowledge about the distribution of molecules in the on state after deactivation, or about the effective pointspread function of the imaging system, was used. Because only linear operations and no deconvolution was applied, we regard the resulting image as consisting of essentially 'raw' data. It should also be noted that these minimal mathematical operations only improve the contrast in the final image but are not required for obtaining the image per se (Supplementary Software).

Conventional wide-field images were recorded before making a stack of RESOLFT subimages. For convenience, pseudowide-field image of the same dimensions can also be calculated by running the analysis without weighting and without subtraction. Summing up all camera images effectively loses information about the deactivation pattern.

Cloning. rsEGFP(N205S) was generated by directed mutagenesis of the rsEGFP (NCBI RefSeq: JQ969017.1) coding sequence using the QuikChange Site Directed Mutagenesis Kit 
(Stratagene). For the creation of fusion constructs with keratin 19, rsEGFP(N205S) was amplified (forward primer, GATCCAC CGGTCGCGGCGTGAGCAAGGGCGAGGAGCTG; reverse primer, ACAACTTAAGAACAACAATTGTTACTTGTACAGC TCGTCCATGCC). The PCR product was cloned into the gateway destination vector pMD-tdEosFP-N using the restriction sites AgeI and AflII to replace the tdEosFP sequence. The plasmids pMD-Ker19-rsEGFP(N205S), coding for the keratin 19-rsEGFP(N205S) fusion, was constructed by a gateway vector conversion (Invitrogen) with pDONR223-Krt19.

Mammalian cell culture. PtK2 cells were cultured in DMEM (Invitrogen) containing 10\% FCS (PAA), 100 units/mL penicillin, $100 \mu \mathrm{g} / \mathrm{mL}$ streptomycin (all from Biochrom) and $1 \mathrm{mM}$ sodium pyruvate (Sigma) at $37^{\circ} \mathrm{C}$ and $5 \% \mathrm{CO}_{2}$. In accordance with the manufacturer's instructions, $5 \times 10^{5}$ cells were transfected with plasmid
DNA using the Nucleofector Technology (Lonza). After $24 \mathrm{~h}$ the growth medium was replaced. Cells were mounted in phenol redfree DMEM (Invitrogen) and imaged $24-72 \mathrm{~h}$ post transfection.

Neuron culture preparation. Cultures of hippocampal neurons were prepared from Wistar rats (both sexes) at postnatal day P0 or P1 in accordance with the regulations of the national Animal Welfare Act and under the approval of local veterinary service. $50 \times 10^{4}$ cells were plated on $100 \mu \mathrm{g} / \mathrm{mL}$ polyornithine-coated (Sigma-Aldrich) and 2\% Matrigel-coated (BD Bioscience) coverslips. Neuronal cultures were maintained in Neurobasal medium (Gibco) supplemented with 2\% B27 serum-free supplement (Gibco), 2 mM L-glutamine (Gibco) and penicillin/streptomycin (Biochrom AG). Cultures were infected on the first day in vitro with a modified Simliki Forest virus containing the pSCA3Lifeact-Dronpa(M159T)GE vector and imaged the day after. 